\title{
Efficient Full-Wave Modeling of Electromagnetic Interference in the Presence of Multiple Non-Collocated Noise Sources
}

\author{
G.-J. Stockman* \\ H. Rogier* \\ D. Vande Ginste*
}

\begin{abstract}
In this contribution a novel method is discussed that is of practical use for analyzing the electromagnetic compatibility behavior of electronic systems. The aim is to develop an efficient technique that mimics radiated immunity and emission tests in the presence of multiple non-collocated noise sources in simulation. The proposed method is simple in that it only relies on the simulated (or measured) radiation pattern of the devices in the system while allowing arbitrary positions. Rotation of the devices is performed by a spherical harmonics decomposition of the radiation patterns together with the application of Wigner-D rotation matrices. The adopted assumption is that the devices are spaced sufficiently far from each other such that there is no coupling via the reactive near-field. The proposed procedure shows good agreement with measurements and full-wave simulations while at the same time it has a low computational cost.
\end{abstract}

\section{Introduction}

Examining the Electromagnetic Compatibility (EMC) behavior of devices in an electronic system is of great importance in today's electronic equipment, not only to pass regulatory and legislative laws concerning the radiated emission and immunity relative to other electronic devices, but also to ensure proper operation of different components internally in such a system. Compliance tests that characterize these properties of a device are performed in an anechoic chamber. In such a test, the device under test is rotated over different angles, while a measurement is performed for every angular position. The disadvantage of this method is that it is costly and time-consuming. Furthermore it can only be performed after devices are already prototyped. It would be benificial to take radiated emission and immunity into account during the design phase (or precompliance phase). This is usually done using simulations, but requires large computational resources and seperate simulations for every angular position.

In this contribution, we develop a formalism that efficiently models the interaction of multiple noncollocated noise sources on another device, based on work performed in [1]. The different devices in the system are allowed to interact via each other's radiative near field. The proposed formalism only relies on the radiation patterns of the devices present in the system and their mutual positions and orientations.

\footnotetext{
${ }^{*}$ IBCN/Electromagnetics Group, Department of Information Technology, Ghent University/iMinds, Sint-Pietersnieuwstraat 41, B9000 Gent, Belgium, e-mail: gert jan.stockman@UGent.be, tel.: +3292643321.
}

\section{Formalism}

Consider the general problem geometry of the case with multiple non-collocated sources $(N)$ and one receiving device in Fig. 1. The devices are represented by their current density sources $\mathbf{j}_{\mathrm{TX}}^{1}\left(\mathbf{r}^{\prime}\right), \cdots, \mathbf{j}_{\mathrm{TX}}^{N}\left(\mathbf{r}^{\prime}\right)$ for the transmitters and $\mathbf{j}_{\mathrm{RX}}\left(\mathbf{r}^{\prime}\right)$ for the receiver. They are defined in volumes $V_{\mathrm{TX}}^{1}, \ldots, V_{\mathrm{TX}}^{N}$ and $V_{\mathrm{RX}}$, respectively, with their appropriate phase centers $\mathcal{O}_{\mathrm{TX}}^{1}, \ldots, \mathcal{O}_{\mathrm{TX}}^{N}$ and $\mathcal{O}_{\mathrm{RX}}$. The electric field generated by the transmitters is

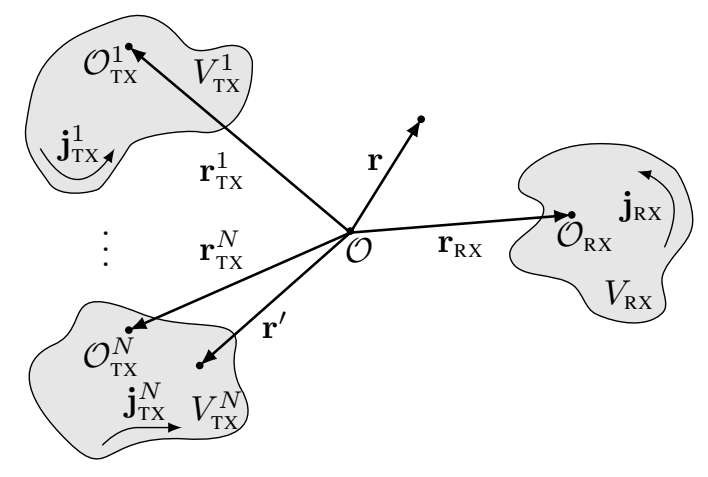

Figure 1: General configuration of interacting devices.

given by the Electric Field Integral Equation (EFIE):

$$
\mathbf{e}(\mathbf{r})=-j \omega \mu \int_{V} \mathcal{G}\left(\mathbf{r}, \mathbf{r}^{\prime}\right) \cdot \mathbf{j}\left(\mathbf{r}^{\prime}\right) \mathrm{d} \mathbf{r}^{\prime},
$$

where $\mathbf{j}\left(\mathbf{r}^{\prime}\right)$ represents the current densities $\mathbf{j}_{\mathrm{TX}}^{1}\left(\mathbf{r}^{\prime}\right), \cdots, \mathbf{j}_{\mathrm{TX}}^{N}\left(\mathbf{r}^{\prime}\right)$ on the transmitters and $\mathbf{j}_{\mathrm{RX}}\left(\mathbf{r}^{\prime}\right)$ on the receiver. $\mu$ is the permeability of the background media, and $\mathcal{G}\left(\mathbf{r}, \mathbf{r}^{\prime}\right)$ the three-dimensional dyadic Green's function. The integration domain $V$ extends over all devices, i.e. $V=\left(V_{\mathrm{TX}}^{1} \cup \cdots \cup V_{\mathrm{TX}}^{N}\right) \cup V_{\mathrm{RX}}$. In this paper we assume that the devices are spaced sufficiently far from each other, such that there is no coupling via the reactive near-field.

For such devices, positioned in each other's Fresnel or Fraunhofer region, the field incident on the receiving device can be accurately approximated by restricting $V$ to $V_{\mathrm{TX}}=\bigcup_{i=1}^{N} V_{\mathrm{TX}}^{i}$ in (1). By applying Gegenbauer's addition theorem [2] to the scalar 3-D Green's function and employing a plane wave expansion, the incoming 
electric field can be written in terms of incoming plane waves and outgoing plane waves:

$$
\begin{aligned}
& \mathbf{e}^{\text {inc }}(\mathbf{r})=-\frac{\omega \mu k}{(4 \pi)^{2}} \sum_{i=1}^{N} \iint_{\Omega} e^{-j \mathbf{k} \cdot\left(\mathbf{r}-\mathbf{r}_{\mathrm{RX}}\right)} T\left(\mathbf{r}_{\mathrm{TX}, \mathrm{RX}}^{i}, \hat{\mathbf{k}}\right) \\
& *[\mathcal{I}-\hat{\mathbf{k}} \hat{\mathbf{k}}] \cdot \int_{V_{\mathrm{TX}}^{i}} e^{j \mathbf{k} \cdot\left(\mathbf{r}^{\prime}-\mathbf{r}_{\mathrm{TX}}^{i}\right)} \mathbf{j}\left(\mathbf{r}^{\prime}\right) \mathrm{d} \mathbf{r}^{\prime} \mathrm{d} \hat{\mathbf{k}}
\end{aligned}
$$

where we defined $\mathbf{r}_{\mathrm{TX}, \mathrm{RX}}^{i}=\mathbf{r}_{\mathrm{RX}}-\mathbf{r}_{\mathrm{TX}}^{i}$. Also, we integrate over the Ewald sphere $\Omega$ and $\mathbf{k}=k(\sin \theta \cos \phi \hat{\mathbf{x}}+$ $\sin \theta \sin \phi \hat{\mathbf{y}}+\cos \theta \hat{\mathbf{z}})$ is the wave vector in spherical coordinates, with $k=\omega \sqrt{\epsilon \mu}$ the wavenumber of the background medium and $\epsilon$ its permittivity. Furthermore, $\mathcal{I}$ is the unit dyadic and $T\left(\mathbf{r}_{\mathrm{TX}, \mathrm{RX}}^{i}, \hat{\mathbf{k}}\right)=\sum_{l=0}^{\infty}(2 l+$ 1) $j^{-l} h_{l}^{(2)}\left(k\left|\mathbf{r}_{\mathrm{TX}, \mathrm{RX}}^{i}\right|\right) P_{l}\left(\hat{\mathbf{k}} \cdot \hat{\mathbf{r}}_{\mathrm{TX}, \mathrm{RX}}^{i}\right)$ the translation operator where $h_{l}^{(2)}(\cdot)$ is the $l$-th order spherical Hankel function of the second kind and $P_{l}(\cdot)$ the Legendre polynomial of degree $l$. The radiation pattern of any transmitting antenna $i$ is defined as

$$
\mathbf{F}_{\mathrm{TX}}^{i}(\hat{\mathbf{k}})=\frac{j \omega \mu}{4 \pi} \hat{\mathbf{k}} \times\left[\hat{\mathbf{k}} \times \int_{V_{\mathrm{TX}}^{i}} e^{j \mathbf{k} \cdot\left(\mathbf{r}^{\prime}-\mathbf{r}_{\mathrm{TX}}^{i}\right)} \mathbf{j}\left(\mathbf{r}^{\prime}\right) \mathrm{d} \mathbf{r}^{\prime}\right],
$$

and similarly for the radiation pattern of the receiver.

Using these, the incident electric field (2) can be written in terms of the radiation patterns of the devices in the system. Assume now, for simplicity, that the transmitters and receiver are one-port devices. Then, an equivalent circuit representation of this configuration is as shown in Fig. 2, where $Z_{\mathrm{TX}}^{i}$ and $Z_{\mathrm{RX}}$ are the radiation impedances of transmitter $i$ and the receiver respectively. Transmitter $i$ is driven by means of a Thévenin generator composed of a sinusoidal voltage source $V_{g}^{i}$ with internal impedance $Z_{g}^{i}$. The Norton equivalent of the receiver consists of a load impedance $Z_{L}$ and a short-circuit current $I_{s c}$. Using the modified expression for the incoming electric field, we obtain a simplified expression for the short-circuit current induced on the receiver by multiple transmitters [3]:

$$
I_{s c}=-\frac{1}{Z} \iint_{\Omega} \sum_{i=1}^{N} T\left(\mathbf{r}_{\mathrm{TX}, \mathrm{RX}}^{i}, \hat{\mathbf{k}}\right) \frac{\mathbf{F}_{\mathrm{TX}}^{i}(\hat{\mathbf{k}})}{V_{0}^{i}} \cdot \mathbf{F}_{\mathrm{RX}}(-\hat{\mathbf{k}}) \mathrm{d} \hat{\mathbf{k}}
$$

where $Z=\sqrt{\frac{\mu}{\epsilon}}$ is the wave impedance of the background medium and $V_{0}^{i}$ the pertinent normalization factor, evolving from the reciprocity theorem, depending on the normalized radiation pattern when operating antenna $i$ is in transmit mode. As the antennas radiation patterns scale with $V_{0}^{i}$, this parameter can be chosen to be $1 \mathrm{~V}$.

In practice, (4) can only be approximated since the infinite sum that occurs in the translation operator has

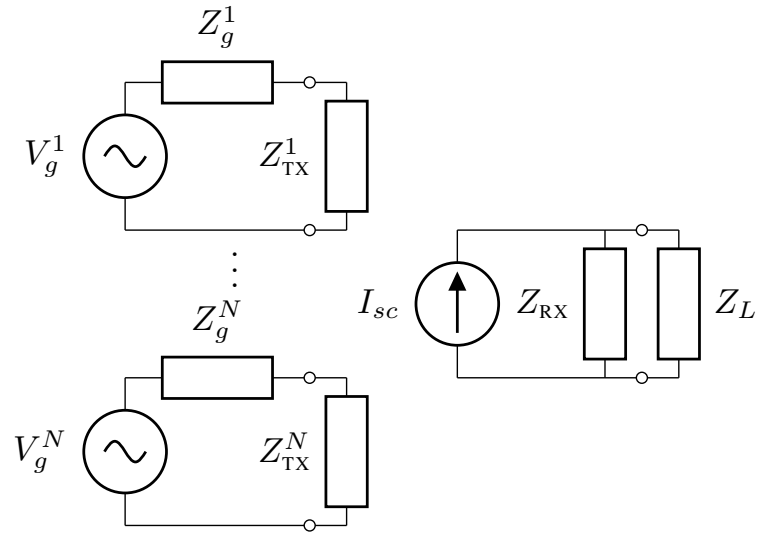

Figure 2: Equivalent circuit of the interaction between devices when there are multiple transmitters.

to be truncated to a finite number of multipoles $L$. How to select this number, and what accuracy it entails is explained in [2].

In summary we can say that in order to calculate the influence of transmitting (noisy) devices on a receiving (susceptible) device, only the measured or simulated radiation patterns of the devices are needed. Furthermore, these devices may be positioned anywhere in space, as long as there is no coupling via the reactive nearfield. This leads to very efficient calculations for multiple transmitter scenarios, in comparison to full-wave solutions, especially when one or more of the devices is repeatedly repositioned in space.

We will now expand the above method into a formalism that is of practical use for the analysis of the EMC behavior of devices and systems. To mimic compliance tests for the assessment of radiated emission or immunity in anechoic chambers, we need the ability to efficiently rotate the devices about their phase center. This can be done by applying the appropriate rotation $\mathcal{R}$ to the radiation patterns of the devices. The rotation is efficiently performed in the spherical harmonics domain $\left(\mathcal{R}_{S H}\right)$, as shown in Fig 3. The transformation to the spherical harmonics domain $\mathcal{F}$ and its inverse $\mathcal{F}^{-1}$ are

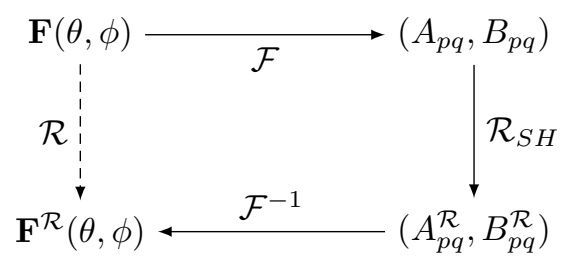

Figure 3: Rotation of $\mathbf{F}(\theta, \phi)$ to $\mathbf{F}^{\mathcal{R}}(\theta, \phi)$ using the spherical harmonics domain. 
given by (5) and (6), respectively:

$$
\begin{aligned}
& \left\{\begin{array}{c}
A_{p q} \\
B_{p q}
\end{array}\right\}=\frac{-1}{p(p+1)} \int_{0}^{2 \pi} \int_{0}^{\pi}\left[q\left\{\begin{array}{c}
j F_{\phi}(\theta, \phi) \\
-F_{\theta}(\theta, \phi)
\end{array}\right\} Y_{p q}^{*}(\theta, \phi)\right. \\
& \left.+\sin \theta\left\{\begin{array}{c}
-F_{\theta}(\theta, \phi) \\
j F_{\phi}(\theta, \phi)
\end{array}\right\} \frac{\mathrm{d} Y_{p q}^{*}(\theta, \phi)}{\mathrm{d} \theta}\right] \mathrm{d} \theta \mathrm{d} \phi \\
& \left\{\begin{array}{l}
F_{\theta}(\theta, \phi) \\
F_{\phi}(\theta, \phi)
\end{array}\right\}=\sum_{p=0}^{P} \sum_{|q| \leq p}\left[\left\{\begin{array}{c}
A_{p q} \\
j B_{p q}
\end{array}\right\} \frac{\mathrm{d} Y_{p q}(\theta, \phi)}{\mathrm{d} \theta}\right. \\
& \left.+\left\{\begin{array}{c}
B_{p q} \\
j A_{p q}
\end{array}\right\} \frac{q Y_{p q}(\theta, \phi)}{\sin \theta}\right]
\end{aligned}
$$

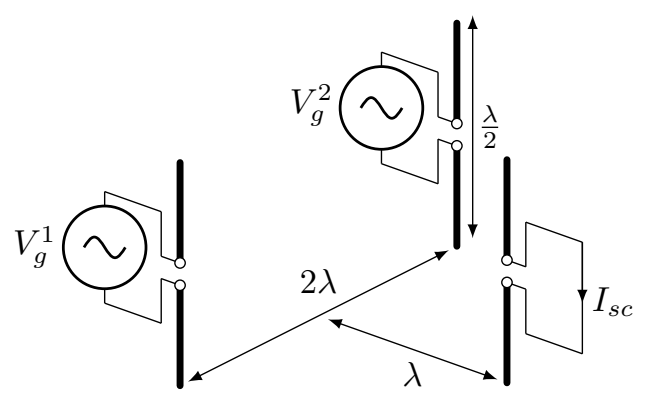

Figure 4: Configuration of the validation example with two transmitters and one receiver.

Here, $Y_{p q}(\theta, \phi)$ are the orthonormalized scalar spherical harmonics and $P$ is a parameter that determines the accuracy. In Fig. 3, the transformation $\mathcal{R}$ represents the desired rotation in the spatial domain. The rotation itself is performed in the spherical harmonics domain by the transformation $\mathcal{R}_{S H}$ that makes use of Wigner Dmatrices. The rotated coefficients of the spherical harmonics expansion are calculated as

$$
\left\{\begin{array}{l}
A_{p q}^{\mathcal{R}} \\
B_{p q}^{\mathcal{R}}
\end{array}\right\}=\left\{\begin{array}{l}
A_{p q} \\
B_{p q}
\end{array}\right\} \sum_{|r| \leq p} e^{-j q \gamma} d_{p q}^{r}(\beta) e^{-j r \alpha},
$$

with $d_{p q}^{r}(\beta)$ the Wigner small d-matrix, given by

$$
\begin{aligned}
& d_{p q}^{r}(\beta)=(-1)^{r-q} \sqrt{(p+r) !(p-r) !(p+q) !(p-q) !} \\
& \cdot \sum_{s}(-1)^{s} \frac{\left(\cos \frac{\beta}{2}\right)^{2(p-s)+q-r}\left(\sin \frac{\beta}{2}\right)^{2 s-q+r}}{(p+q-s) ! s !(r-q+s) !(p-r-s) !}
\end{aligned}
$$

Here, the range of $s$ is determined by the condition that all factorials are nonnegative, thus $s \in$ $[\max (0, q-r), \min (p+q, p-r)]$. In (7)-(8), $\alpha, \beta$ and $\gamma$ are the standard Euler angles that define the rotation using the $z-y-z$ convention in a right-handed frame. The Euler angles $(\alpha, \beta, \gamma)$ are readily related to the desired inclination and azimuthal angles $\theta$ and $\phi$, by choosing $\alpha=\phi, \beta=\theta$ and $\gamma=0$.

\section{Validation Example}

In order to validate the formalism, a numerical experiment is performed with half-wavelength dipole antennas. In this example, as shown in Fig. 4, two transmitters are placed at positions $\mathbf{r}_{\mathrm{TX}}^{1}=\lambda \hat{\mathbf{x}}$ and $\mathbf{r}_{\mathrm{TX}}^{2}=-\lambda \hat{\mathbf{x}}$ while the receiver is located at $\mathbf{r}_{\mathrm{RX}}=\lambda \hat{\mathbf{y}}$. The shortcircuit current $I_{s c}$ obtained with our method as shown in (4) is compared to the Method of Moments (MoM) reference solution for arbitrary thin wires [4]. The wire thickness used in this example equals $10^{-4} \lambda$ and the MoM simulation uses five segments to model one dipole. The example in Fig. 4 is comparable to the equivalent circuit shown in Fig. 2 with $N=2$ and $Z_{g}^{1}=Z_{g}^{2}=Z_{L}=0 \Omega$. The excitation voltages between the terminals of the transmitters are chosen $V_{g}^{1}=V_{g}^{2}=1 \mathrm{~V}$. In the MoM simulation, the shortcircuit current is calculated directly by inverting the full MoM matrix equation.

To verify our novel method, with rotations performed using spherical harmonics, we start from the dipole's radiation pattern $\mathbf{F}(\theta, \phi)$. This radiation pattern is obtained via a MoM simulation for a single dipole. This radiation pattern is decomposed into spherical harmonics by (5) and afterwards rotated using (7). Subsequently, the rotated radiation patterns $\mathbf{F}^{\mathcal{R}}(\theta, \phi)$ are reconstructed using (6) and finally substituted into (4) to calculate the short-circuit current $I_{s c}$. For this example we rotate the recieving dipole over Euler angles $\alpha=\beta=\gamma$, varying from 0 till $2 \pi$ for which we use $P=L=2$. A comparison between the short-circuit current obtained from the MoM simulation and the novel spherical harmonics method is given in Fig. 5, where an excellent agreement is observed. The

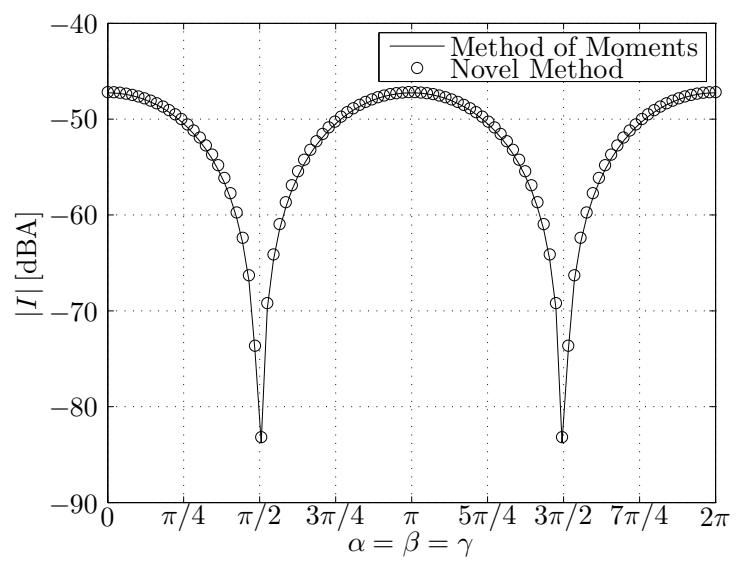

Figure 5: Amplitude of the short-circuit current at the port of the receiving dipole. The receiving dipole is rotated over Euler angles $\alpha=\beta=\gamma$. 
MoM simulation over all angles took $84.73 \mathrm{~s}$ whereas the novel method only took $1.63 \mathrm{~s}$. Hence, an impressive speed-up factor of about $50 \times$ is achieved. In addition to the much shorter simulation times, only the $(P+1)^{2}$ coefficients $A_{p q}$ and $B_{p q}$ have to be stored to simulate any situation with relative position and orientation. All simulations have been carried out on an Intel $\mathrm{R}$ Core $^{\mathrm{TM}} \mathrm{i} 7-2600$ processor running at $3.40 \mathrm{GHz}$ and with $16 \mathrm{~GB}$ of memory.

\section{Application Example}

To demonstrate the practicability of the developed formalism, a measurement has been performed in an anechoic chamber. In this example, a Standard Gain Horn (SGH) acts as the receiver, while two microstrip patch antennas act as two non-collocated noise sources. The measurement setup is shown in Fig. 6. The two microstrip patch antennas are placed symmetrically around the $\mathrm{SGH}$, and are rotated about $68^{\circ}$ towards the $\mathrm{SGH}$. The distance between the two phase centers of the patch antennas is $d_{\text {patch-patch }}=2.55 \mathrm{~m}$, while the distance between the phase center of a patch antenna and the phase center of the SGH equals $d_{\text {patch-SGH }}=$ $3.45 \mathrm{~m}$. During the measurement, the polarisation of

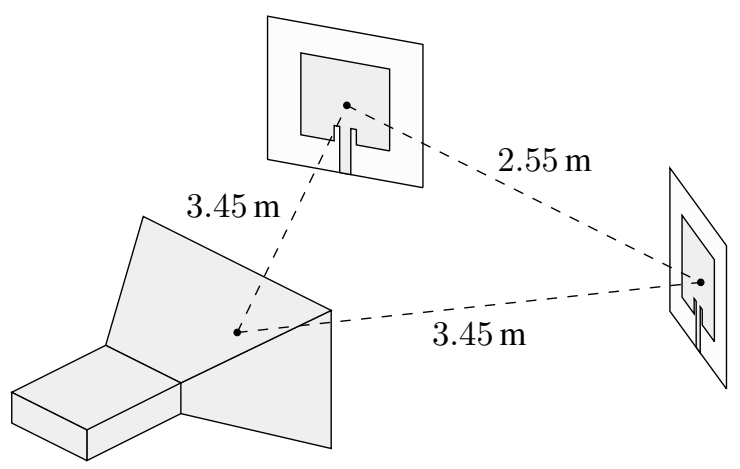

Figure 6: Measurement setup where a SGH is influenced by two patch antennas acting as non-collocated noise sources.

the transmitter is altered by rotating the SGH around the axis of its main beam from $-\pi$ to $\pi$. For every orientation, the scattering parameters between the antennas are measured for comparison with simulation. The SGH and patch antennas are all designed to radiate at $1.5 \mathrm{GHz}$. In simulation, the rotation is performed using spherical harmonics and Wigner-D rotation matrices. The radiation patterns of the SGH and patch antennas are obtained from an analytical solution and a simulation with the 3-D planar full wave solver Momentum from Advanced Design System (ADS) of Keysight Technologies, respectively. The obtained link between one of the patch antennas and the SGH is shown in Fig. 7, where $P=L=5$. An excellent agreement

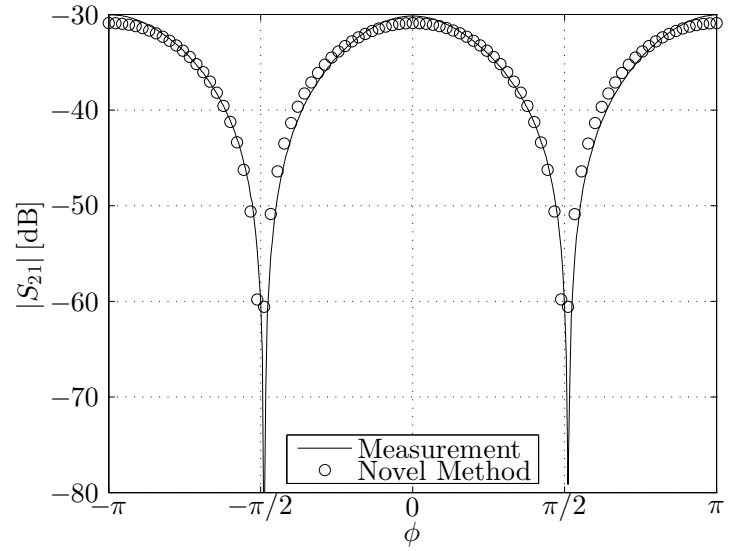

Figure 7: The measured and simulated $(P=L=5)$ link between one of the noise sources and the SGH. The SGH is rotated over $-\pi \leq \phi \leq \pi$.

between measurement and simulation is obtained. For $\phi=0$ the difference between measurement and simulation is even smaller than $1 \mathrm{~dB}$.

\section{Conclusions}

In this contribution we have presented a method that allows to determine the electromagnetic interference induced on a device in the presence of multiple noncollocated noise sources. The method solely relies on a single measurement or simulation of the radiation patterns of the devices in the system. Afterwards, the devices can, as long as there is no coupling via the reactive near-field, be positioned anywhere in space and given an arbitrary orientation without requiring completely new simulations, reducing the computational complexity of our method as compared to a full-wave solver.

\section{References}

[1] G.-J. Stockman, H. Rogier, and D. Vande Ginste, "Efficient modeling of interactions between radiating devices with arbitrary relative positions and orientations," IEEE Transactions on EMC, vol. 56, no. 6, pp. 1313-1321, Dec 2014.

[2] W. C. Chew, J.-M. Jin, E. Michielssen, and J. Song, Fast and efficient algorithms in computational electromagnetics. Artech House Publishers, 2001.

[3] J. Van Bladel, "On the equivalent circuit of a receiving antenna," IEEE Antennas and Propagation Magazine, vol. 44, no. 1, pp. 164-165, February 2002.

[4] W. C. Gibson, The Method of Moments in Electromagnetics. Chapman \& Hall/CRC, 2008, ch. 4, pp. 73-79. 